\title{
Functional outcomes of posterior intravaginal slingplasty: report on its impact on urinary, bowel and psychosexual function
}

\author{
R. Oliver $\cdot$ O. Odutola $\cdot$ A. Coker
}

Received: 12 January 2008/Accepted: 19 March 2008/Published online: 19 April 2008

(C) Springer-Verlag 2008

\begin{abstract}
The functional impact of any clinical intervention should be the ultimate arbiter of the success of such interventions. This study evaluated the outcomes of urinary, bowel and psychosexual functions in 31 patients undergoing posterior vaginal slingplasty for pelvic organ prolapse. The results show significant improvement in all prolapse symptoms. Urinary symptoms of overactive bladder and stress incontinence improved significantly, as well as the bowel symptoms of obstructed defecation and urgency. Sexual function and psychological state also improved significantly with the procedure. Significantly, to date, this is the first study assessing the psychological impact of pelvic organ prolapse and the remission of these symptoms with the slingplasty. The posterior vaginal slingplasty is an effective procedure in prolapse correction, along with alleviation of the majority of bothersome symptoms caused by the prolapse.
\end{abstract}

Keywords Prolapse surgery · Posterior slingplasty

\section{Introduction}

Pelvic organ prolapse has a lifetime prevalence risk of 30 $50 \%$ and an $11 \%$ lifetime risk for surgery, accounting for $20 \%$ of women on the waiting list for major gynaecological surgery in the UK. It is well established that the functional impact of any surgical procedure on the patient should be the final measure of success and not measurements of

R. Oliver $(\bowtie) \cdot$ O. Odutola $\cdot$ A. Coker

Queens Hospital,

Rom Valley Way,

Romford, Essex RM7 0AG, UK

e-mail: oliverreebs@aol.com morbidity, mortality and anatomical correction alone. The posterior vaginal slingplasty is effective in achieving the anatomic correction of pelvic organ prolapse and has been widely used since its inception ten years ago by Petros [1] Most of the surgical procedures for prolapse correction have been evaluated for functional outcomes, but, to date, very few studies have specifically evaluated the functional outcomes of posterior slingplasty. de Tayrac et al. recently assessed the functional outcomes of slingplasty as part of a randomised trial comparing slingplasty with sacrospinous suspension and found both procedures to afford significant functional improvement [2]. The assessment of functional symptoms is of paramount importance, as only a weak to moderate correlation has been established between the degree of prolapse and symptoms complained of by the patient.

The negative impact of a prolapse on urinary, bowel and sexual function has been well established and urinary symptoms have been recorded as the most commonly occurring symptom followed by symptoms attributable to the prolapse itself. Psychiatric and psychological symptoms can arise or contribute to a disease process and the assessment of these cannot be discounted if effectiveness of the operative procedure is to be evaluated accurately. There is evidence that psychological disorders play an important role in disease process, depression being the fourth leading cause of disease burden, accounting for $4.4 \%$ of the total disability-adjusted life years in the world in 2000 [3]. In our study, we have analysed the psychological impact of a prolapse and the impact of surgical procedures on these symptoms.

All of the symptom evaluation has been performed using validated questionnaires, the paper version of the electronic pelvic floor assessment questionnaire (e-PAQ) [4] and the General Health Questionnaire-30 (GHQ-30) [5]. The use of 
questionnaires that are validated is important, as they have been shown to be reliable in assessing symptoms and are reproducible. Both of the above questionnaires have been validated as to their content and criterion validity, reliability and responsiveness. The use of validated questionnaires in our study strengthens the reliability and significance of our findings.

The primary aim of this study is to evaluate all of the important functional outcomes of a pelvic organ prolapse corrective surgery, i.e. prolapse symptoms, urinary and bowel symptoms, and also psychological and sexual functions.

\section{Methods}

All women who had an intravaginal slingplasty procedure performed for pelvic organ prolapse in a minimal access gynaecology surgical unit in the United Kingdom were assessed as to their functional outcomes before and after the procedure. Forty-six women were operated upon by a single surgeon over a period of three years.

\section{Operative technique}

The aim of the technique is to repair the three levels of vaginal support described by DeLancey [6]. The operative procedure has been described for vault prolapse with modifications for uterovaginal prolapse in our previous series of 14 patients [7].

The technique reinforces the uterosacral ligament by placing polypropylene tape between the perineum and the vaginal vault. A vertical incision is made in the vault and the IVS Tunneller (Tyco Health Care, USA) is used to place polypropylene tape as an inverted ' $\mathrm{U}$ ' around the rectum and attach it behind the posterior vaginal wall. The tip of the tunneller is elevated and inserted into the ischiorectal fossa and through the rectovaginal fascia and into the vaginal incision. The tip of the tunnelling device is guided by a finger positioned in the vaginal incision. Vaginal and rectal examinations are performed before and after the insertion to ensure correct placement of the tape and to exclude damage to the rectum. The apical portion of the tape is anchored to each corner of the vault at the estimated insertion site of the uterosacral ligament [7].

At the pre- and postoperative appointments, the degree of prolapse was assessed using the Baden-Walker vaginal prolapse grade. The Baden-Walker system was preferred due to long familiarity of usage and its proven interexaminer reliability [8].

The e-PAQ was used to ascertain the symptoms attributable primarily to the prolapse. It was also used to ascertain the bladder, bowel and sexual function preoper- atively and postoperatively. The e-PAQ evaluates the impact of a prolapse on bladder, bowel, vagina and sexual function using a four-point ordinal scale of never, occasionally, most of the time and all of the time [4]. In the urinary dimension, it tests the following domains of voiding: pain and sensation, overactive bladder symptoms, stress urinary symptoms and quality of life. The domains of irritable bowel symptoms, constipation, evacuation, continence and quality of life are tested in the bowel dimension. Vaginal symptoms of pain, prolapse and quality of life are tested and in the sexual dimension, the domains of overall sexual function and the effect of bladder, bowel and vaginal problems on sex is tested. All of the above domains are scored from ' $0=$ best possible' to ' $100=$ worst possible' and are converted into a final score. Additionally, each domain has a secondary scale assessing the impact or the 'bother' on a four-point scale of 'not a problem,' 'a bit of a problem,' 'quite a problem' and 'a serious problem.' The content, construct and criterion validity, internal consistency, reliability and responsiveness of the questionnaire have been proven to be effective in assessing the impact of pelvic organ prolapse. It is also effective in comparing treatment efficacy, as it has been shown to be sensitive to the change of symptoms following surgical intervention.

The psychological outcomes were measured using the GHQ-30 questionnaire, both preoperatively and postoperatively. The GHQ-30 is a screening instrument designed to detect current psychiatric disorder and is designed to be self-administered. The 30 -item questionnaire contains five factors, namely, anxiety, depression, social dysfunction, feelings of incompetence and difficulty in coping. The GHQ-30 has been widely used in many countries for detecting psychological morbidity and has been extensively validated by 29 studies [5]. The GHQ-30 questionnaire was scored using the GHQ scoring $0,0,1,1$ from left to right for the four options to each of the 30 questions, giving a maximum score of 30 . A threshold score of four or more was used to identify respondents with a possible psychiatric disorder, more than 15 is evidence of distress and more than 20 suggests severe problems and psychological distress.

The primary outcome measure was the change of the above symptoms of bladder, bowel, sexual and psychological function in the postoperative period.

The preoperative and the postoperative scores were compared and ranked to analyse the change of the functional vaginal, bladder, bowel and urinary symptoms from the preoperative to the postoperative period. Comparison was performed using the Wilcoxon signed-rank test, as the data was non parametric. Statistical analysis was performed using the SigmaStat 3.5 statistical programme (Systat Inc.) and, where appropriate, statistical advice was sought. 
The questionnaires were used as part of the clinical assessment of the patient, as well as the assessment of the surgical procedure as part of establishing the effectiveness of clinical practice. Written consent was also obtained from all of the patients for the use of their data for scientific publication. Ethics committee approval was obtained for the use and publication of the data from the questionnaires.

\section{Results}

The e-PAQ and the GHQ-30 questionnaires were completed both preoperatively and postoperatively by 31 of the 46 patients who had posterior slingplasty performed. Fifteen of the 46 patients were not contactable, as the follow up period was lengthy in some cases. The mean age was 68 years (standard deviation $[\mathrm{SD}]=9.83$ ), with a range of 52-90 years. Only two of the patients were premenopausal and the rest $(n=29)$ were postmenopausal. The postoperative follow up period ranged from 6 months to 42 months (mean=19 months; $\mathrm{SD}=10.29$ ).

\section{Concomitant procedures}

All patients had the IVS tape inserted as described above. Additionally, 24 (52\%) patients had concomitant procedures performed. Twelve of the 24 patients with concomitant procedures had an additional pelvic floor repair procedure (cystocele repair in two, rectocele repair in five and combined repairs in five). Twelve patients had vaginal hysterectomies performed prior to the IVS insertion, as, during assessment under anaesthesia, it was felt that the degree of uterovaginal prolapses in these patients were of such a degree as to potentially compromise the success of the repair.

There were no significant postoperative complications.

Both questionnaires were scored and analysed as detailed above. Table 1 shows the change in symptoms attributable to the prolapse and also in bladder, bowel and sexual functions after the surgical procedure. Additionally, impact on the change in the psychological status is also detailed.

\section{Vaginal symptoms}

All of the patients had awareness of a lump in the vagina preoperatively, which was cured in $100 \%$ of cases after the procedure $(p \leq 0.001)$. There was no relapse of this cardinal symptom noted by any of the patients postoperatively and the regression of the impact of the prolapse was also significant $(p \leq 0.001)$. The other symptom attributable to the prolapse that was assessed was pain, which was present in $94 \%$ of the patients $(n=29)$. Pain and the impact of pain showed significant regression postoperatively $(p \leq 0.001)$. The quality of life due to the vaginal symptoms also improved significantly $(p \leq 0.001)$ (Table 1$)$.

\section{Bladder symptoms}

There was a statistically significant improvement in the symptoms of overactive bladder and stress incontinence between the preoperative and postoperative scores (Table 1). The most common symptoms were overactive bladder symptoms $(n=29 ; 93.54 \%) ; 18$ out of the 29 patients with preoperative symptoms reported an improvement $(p=0.014)$. Twenty nine percent $(n=9)$ of the patients reported their overactive symptoms to be unaffected by the slingplasty. In line with the results for overactive bladder, stress incontinence improved in 17 out of the 25 patients who had preoperative symptoms $(p=0.002)$. Thirty two percent $(n=10)$ of the patients reported no improvement in their stress incontinence after surgery. The impact of the above symptoms on the patients and their quality of life $(p=0.003)$ improved significantly postoperatively. Although there was no significant improvement in the symptom of voiding difficulty, the impact of that symptom on the patient improved significantly postoperatively $(p=0.014)$.

\section{Bowel symptoms}

Analysis showed a statistically significant improvement in irritable bowel symptoms and evacuation difficulty (Table 1). Irritable bowel symptoms was the second most common symptom reported preoperatively $(n=30 ; 96.77 \%) ; 15$ of the 30 patients with preoperative symptoms reported an improvement $(p=0.047)$ and in 14 patients, the symptom worsened after the surgery. Two $(6.45 \%)$ patients reported irritable bowel symptoms to be unaffected by the slingplasty. Twenty of the 29 patients with preoperative symptoms reported significant improvement $(p=0.014)$. Although there was no statistically significant improvement in faecal incontinence and constipation symptoms, the impact of faecal incontinence on the patient showed significant improvement ( $p=0.005)$, as did the quality of life attributable to the bowel symptoms.

\section{Sexual function}

In our series, only ten patients out of the 31 were sexually active and all sexually active patients reported sexual dysfunction in relation to their prolapse. The 21 remaining patients who were not sexually active preoperatively did not commence sexual activity postoperatively. Analysis was based on the sexually active patients and showed a statistically significant improvement in the overall sexual 
Table 1 Change of bladder, bowel, vaginal, sexual and psychological symptoms from the preoperative to the postoperative period

\begin{tabular}{|c|c|c|c|c|c|c|}
\hline DOMAIN & $\begin{array}{l}\text { Preoperative } \\
\text { symptoms/impact }\end{array}$ & $\begin{array}{l}\text { No preoperative } \\
\text { symptoms/impact }\end{array}$ & Symptoms better & Symptoms worse & $\begin{array}{l}\text { No change } \\
\text { in symptoms }\end{array}$ & $p^{*}$ \\
\hline \multicolumn{7}{|l|}{ Urinary $(n=31)$} \\
\hline Voiding & $21(67.74 \%)$ & $10(32.2 \%)$ & $16(51.61 \%)$ & $8(25.8 \%)$ & $7(22.58 \%)$ & 0.120 \\
\hline Impact of voiding & $19(61.29 \%)$ & $12(38.70 \%)$ & $15(48.38 \%)$ & $3(9.67 \%)$ & $13(41.93 \%)$ & 0.014 \\
\hline Pain and sensation & $20(64.51 \%)$ & $11(35.48 \%)$ & $12(38.70 \%)$ & $6(19.35 \%)$ & $13(41.93 \%)$ & 0.189 \\
\hline Impact of pain and sensation & $16(51.61 \%)$ & $15(48.38 \%)$ & $10(32.2 \%)$ & $5(16.12 \%)$ & $16(51.61 \%)$ & 0.122 \\
\hline Overactive bladder & $29(93.54 \%)$ & $2(6.45 \%)$ & $18(58.06 \%)$ & $4(12.90 \%)$ & $9(29.03 \%)$ & 0.014 \\
\hline Impact of overactive bladder & $25(80.64 \%)$ & $6(19.35 \%)$ & $17(54.83 \%)$ & $3(9.67 \%)$ & $11(35.48 \%)$ & 0.004 \\
\hline Stress urinary incontinence & $25(80.64 \%)$ & $6(19.35 \%)$ & $17(54.83 \%)$ & $4(12.90 \%)$ & $10(32.2 \%)$ & 0.002 \\
\hline Impact of stress incontinence & $22(70.96 \%)$ & $9(29.03 \%)$ & $13(41.93 \%)$ & $2(6.45 \%)$ & $16(51.61 \%)$ & 0.005 \\
\hline Quality of life & $24(77.41 \%)$ & $7(22.5 \%)$ & $19(61.29 \%)$ & $1(3.22 \%)$ & $11(35.48 \%)$ & 0.003 \\
\hline \multicolumn{7}{|l|}{ Bowel $(n=31)$} \\
\hline Irritable bowel symptoms & $30(96.77 \%)$ & $1(3.22 \%)$ & $15(48.38 \%)$ & $2(6.45 \%)$ & $14(45.16 \%)$ & 0.047 \\
\hline Constipation & $18(58.06 \%)$ & $13(41.93 \%)$ & $12(38.7 \%)$ & $3(9.67 \%)$ & $16(51.61 \%)$ & 0.063 \\
\hline Evacuation & $29(93.54 \%)$ & $2(6.45 \%)$ & $20(64.51 \%)$ & $4(12.90 \%)$ & $7(22.58 \%)$ & 0.014 \\
\hline Impact of evacuation & $26(83.87 \%)$ & $5(16.12 \%)$ & $13(41.93 \%)$ & $2(6.45 \%)$ & $16(51.61 \%)$ & 0.005 \\
\hline Continence & $31(100 \%)$ & 0 & $19(61.29 \%)$ & $2(6.45 \%)$ & $10(32.2 \%)$ & 0.051 \\
\hline Impact of continence & $24(77.41 \%)$ & $7(22.58 \%)$ & $14(45.16 \%)$ & $1(3.22 \%)$ & $16(51.61 \%)$ & 0.005 \\
\hline Quality of life & $16(51.61 \%)$ & $15(48.38 \%)$ & $14(45.16 \%)$ & $3(9.67 \%)$ & $14(45.16 \%)$ & 0.013 \\
\hline \multicolumn{7}{|l|}{ Vagina $(n=31)$} \\
\hline Pain & $29(93.54 \%)$ & $2(6.45 \%)$ & $25(80.64 \%)$ & $3(9.67 \%)$ & $3(9.67 \%)$ & $<0.001$ \\
\hline Impact of pain & $25(80.64 \%)$ & $6(19.35 \%)$ & $21(67.74 \%)$ & $1(3.22 \%)$ & $9(29.03 \%)$ & $<0.001$ \\
\hline Prolapse & $31(100 \%)$ & 0 & $31(100 \%)$ & 0 & 0 & $<0.001$ \\
\hline Impact of prolapse & $29(93.54 \%)$ & $2(6.45 \%)$ & $29(93.54 \%)$ & 0 & $2(6.45 \%)$ & $<0.001$ \\
\hline Quality of life & $29(93.54 \%)$ & $2(6.45 \%)$ & $27(87.09 \%)$ & $1(3.22 \%)$ & $3(9.67 \%)$ & $<0.001$ \\
\hline \multicolumn{7}{|l|}{$\operatorname{Sex}^{\mathrm{a}}(n=10)$} \\
\hline Sex overall & $10(100 \%)$ & 0 & $8(80 \%)$ & 0 & $2(20 \%)$ & 0.004 \\
\hline Sex and bladder & $10(100 \%)$ & 0 & $10(100 \%)$ & 0 & 0 & 0.002 \\
\hline Impact of sex and bladder & $9(90 \%)$ & $1(10 \%)$ & $8(80 \%)$ & 0 & $2(20 \%)$ & 0.002 \\
\hline Sex and bowel & $6(60 \%)$ & $4(40 \%)$ & $6(60 \%)$ & $1(10 \%)$ & $3(30 \%)$ & 0.096 \\
\hline Impact of sex and bowel & $4(40 \%)$ & $6(60 \%)$ & $4(40 \%)$ & 0 & $6(60 \%)$ & 0.099 \\
\hline Sex and vagina & $9(90 \%)$ & $1(10 \%)$ & $9(90 \%)$ & 0 & $1(10 \%)$ & 0.007 \\
\hline Impact of sex and vagina & $9(90 \%)$ & $1(10 \%)$ & $8(80 \%)$ & 0 & $2(20 \%)$ & 0.002 \\
\hline Psychological status & $30(96.77 \%)$ & $1(3.22 \%)$ & $29(93.54 \%)$ & 0 & $2(6.45 \%)$ & $<0.001$ \\
\hline
\end{tabular}

Data expressed as $n(\%)$

*Wilcoxon signed rank test

${ }^{a}$ Analysed on sexually active patients $(n=10)$

activity and the effect of bladder and vaginal symptoms on sexual activity (Table 1$)$. Eighty percent $(n=8)$ of patients reported improvement in overall sexual function and $20 \%$ $(n=2)$ reported no change. The impact of these symptoms on the patients also lessened. The majority of the patients improved in the frequency and satisfaction of sexual activity and avoided sexual activity less after the slingplasty.

\section{Psychological status}

Improvements in psychological status with the surgical procedure on the GHQ-30 scores were statistically significant $(p \leq 0.001)$. All 29 patients who reported scores of more than 4 showed significant improvement and the rest reported no change with the procedure.
Preoperatively, 15 (48.38\%) patients had scores of more than 15 , showing evidence of distress, and this number showed a significant decrease to three $(9.67 \%)$ patients postoperatively. The number of patients with scores of more than 20 suggesting severe problems and psychological distress were six $(19.35 \%)$ preoperatively and this decreased to one $(3.22 \%)$ patient after the slingplasty.

Physical examination

There was a significant reduction in the seven patients who presented with a cystocele (median reduction from Grade 2 to Grade 0$)(p \leq 0.001)$. Similar results were seen with the rectocele (median reduction from Grade 2 to Grade 0 ) ( $p \leq$ 0.001 ) and with the vault and uterovaginal prolapse 
reduction (median reduction from Grade 3 to Grade 0 ) ( $p \leq$ $0.001)$. No recurrence was seen in the follow up period (mean=19 months) (Table 2).

\section{Discussion}

An assessment of the functional outcomes as the final determinant of the success of slingplasty is essential. The study by de Tayrac et al. addressed the functional outcomes in comparison to the sacrospinous fixation and found similar remissions of the functional symptoms [2]. This is the first study, to our knowledge, addressing the functional outcomes of the posterior vaginal slingplasty exclusively, especially the psychological symptoms, which are debilitating to the patient. The complete remission of the main prolapse symptom of feeling a lump in the vagina in all of the patients is significant in gauging the effectiveness of the procedure. The postoperative follow up period was on an average of 19 months, with a maximum period of 42 months. The postoperative examination confirmed the absence of any recurrence of the prolapse during the follow up period. The absence of any relapse noted by the patients in this time period is again significant, and bears evidence to the postoperative examination findings. Our study also confirms the significant remission in vaginal pain and soreness. The remission in the latter symptom could be explained due to the anatomical repositioning of the laxity in the posterior compartment achieved by the slingplasty.

In contrast to the effect of the majority of surgical procedures for prolapse, stress incontinence improved significantly after the slingplasty. This contradicts the widely held belief that the correction of a prolapse could unmask stress incontinence and would aid the argument that the posterior slingplasty buttresses all three levels of vaginal support. The improvement in stress urinary incontinence could serve as a distinct advantage of this procedure compared to other surgical corrective procedures for prolapses, where concomitant colposuspension has been advocated to prevent significant postoperative stress incontinence [9]. Overactive bladder symptoms was the most common bladder symptom to show significant improvement. This finding is in line with recent studies which have established the reduction in overactive bladder symptoms achieved by vaginal corrective surgery for prolapses [10].

The anatomical correction by the posterior sling achieves realignment of the anorectal angle and this could be instrumental in the remission of the bowel symptoms causing difficulty in evacuation. Although the improvement in faecal incontinence did not reach statistical significance, it is noteworthy that the impact of the symptom on the patient improved significantly.

The significant improvement in sexual function could be the cardinal reason for the increased patient satisfaction with the procedure. There is nil or minimal excision of vaginal skin and, hence, a low incidence of dyspareunia. The increased frequency of and satisfaction with intercourse reflects good anatomical correction and maintenance of vaginal length and anatomy.

Most of the patients in our series had high GHQ scores, indicating psychological distress. This highlights the significant but often overlooked impact of a prolapse on general well being. It is pertinent that $97 \%$ of the patients with preoperative psychological symptoms improved after the surgery and this lays emphasis on the importance of conducting studies assessing functional impacts.

Important questions remain pertaining to the long-term success of these symptom remissions and cure. Do they recur and, if so, is the recurrence accompanied by the relapse of the prolapse correction? Continuing follow up of these patients is required to establish the long-term effectiveness of this procedure in achieving anatomical correctness, including the impact on the functional symptoms.

\section{Conclusion}

The posterior vaginal slingplasty is a minimally access procedure achieving anatomical correction of a prolapse and has been shown, in our study, to improve vaginal, urinary, bowel, sexual and psychological symptoms. The improvements in these functional symptoms are significant and are highly positive determinants of the final success of this procedure. Further long-term, larger studies are required to establish the durability of these symptom remissions, as well their impact on the quality of life.
Table 2 Results of postoperative evaluation

*Baden-Walker grading system

${ }^{\text {a }}$ Mann-Whitney rank sum test

\begin{tabular}{llllr}
\hline Prolapse & $\begin{array}{l}n=\text { no. of } \\
\text { patients }\end{array}$ & $\begin{array}{l}\text { Preoperative } \\
\text { grade* (median) }\end{array}$ & $\begin{array}{l}\text { Postoperative } \\
\text { grade* (median) }\end{array}$ & $p$-value $^{\text {a }}$ \\
\hline Cystocele & 7 & 2 & 0 & $p \leq 0.001$ \\
Rectocele & 10 & 2 & 0 & $p \leq 0.001$ \\
Vault/uterovaginal & 46 & 3 & 0 & $p \leq 0.001$ \\
prolapse & & & & \\
\hline
\end{tabular}


Acknowledgements We gratefully acknowledge Mr. Stephen Radley for his permission and support in using the paper version of the e-PAQ. We also acknowledge NFer-Nelson for granting us permission to use the GHQ-30 questionnaire.

Ethical declaration This manuscript complies with the current ethical laws in the United Kingdom.

Disclosure of interests None of the authors have any disclosable interests.

Funding No declarations of funding exist.

\section{References}

1. Petros PE (1997) New ambulatory surgical methods using an anatomical classification of urinary dysfunction improve stress, urge and abnormal emptying. Int Urogynecol J Pelvic Floor Dysfunct 8:270-277

2. de Tayrac R, Mathé ML, Bader G, Deffieux X, Fazel A, Fernandez H (2008) Infracoccygeal sacropexy or sacrospinous suspension for uterine or vaginal vault prolapse. Int $\mathrm{J}$ Gynaecol Obstet 100(2):154-159

3. Ustün TB, Ayuso-Mateos JL, Chatterji S, Mathers C, Murray CJ (2004) Global burden of depressive disorders in the year 2000. Br J Psychiatry 184:386-392

4. Bradshaw HD, Hiller L, Radley S, Farkas AG, Radley SC (2006) Development and psychometric testing of a symptom index for pelvic organ prolapse. J Obstet Gynaecol 26(3):241-252

5. Goldberg DP (1972) The detection of psychiatric illness by questionnaire. Oxford University Press, London

6. DeLancey JOL (1992) Anatomic aspects of vaginal eversion after hysterectomy. Am J Obstet Gynecol 166:1717-1728

7. Oliver R, Dasgupta C, Coker A (2006) Posterior intravaginal slingplasty for vault and uterovaginal prolapse: an initial experience. Gynecol Surg 3:88-92

8. Baden WF, Walker TA (1992) Surgical repair of vaginal defects. Lippincott, Philadelphia, PA

9. Brubaker L, Cundiff GW, Fine P, Nygaard I, Ritcher HE, Visco AG, Zyczynski H, Brown MB, Weber AM; Pelvic Floor Disorders Network (2006) Abdominal sacrocolpopexy with Burch colposuspension to reduce urinary stress incontinence. N Eng J Med 354(15):1557-1566

10. Foster RT Sr, Barber MD, Parasio MF, Walters MD, Weidner AC, Amundsen CL (2007) A prospective assessment of overactive bladder symptoms in a cohort of elderly women who underwent transvaginal surgery for advanced pelvic organ prolapse. Am J Obstet Gynecol 197(1):82.e1-82.e4 